Secretaria de Estado de Saúde de Minas Gerais (SES-MG) - Belo Horizonte (MG), Brasil.

ana.mendes@saude.

mg.gov.br

\section{A vigilância em saúde ambiental como resposta ao desastre do rompimento da barragem de rejeitos em Brumadinho}

\author{
Environmental health surveillance as a response to the tailing dam \\ disruption disaster in Brumadinho
}

Ana Paula Mendes Carvalho', Gabriela Lopes Marques', Joice Rodrigues da Cunha', Rosiane

Aparecida Pereira', Talita Silva de Oliveira'

DOI: $10.1590 / 0103-11042020 E 225$

RESUMO A saúde ambiental se constitui o campo da saúde pública responsável pelas políticas públicas relacionadas com a interação entre a saúde humana e os fatores do meio ambiente. A gestão de risco de desastres envolve um processo de antecipação, planejamento e preparação para resposta, com atuação de diferentes áreas de governo, como o setor saúde. Este relato de experiência teve o objetivo de descrever as ações desenvolvidas pela vigilância em saúde ambiental da Secretaria de Estado de Saúde de Minas Gerais em resposta ao rompimento da barragem B1 em Brumadinho. Entre as ações desenvolvidas, destacam-se o monitoramento da qualidade da água para consumo humano nos municípios atingidos, a participação em reuniões em espaços de decisão e deliberação e a produção de documentos técnicos. As experiências vivenciadas pela equipe, durante as ações de resposta ao desastre, motivaram reflexões sobre a necessidade de fortalecer o desenvolvimento das ações de forma intra e intersetorial e a importância de qualificação dos dados registrados nos sistemas de informação e de conhecimento das informações locais de cada território, a fim de subsidiar a atuação do setor saúde de modo oportuno em situações de desastre.

PALAVRAS-CHAVE Vigilância em saúde pública. Desastres provocados pelo homem. Saúde ambiental.

\begin{abstract}
Environmental health is the field of public health responsible for public policies related to the interaction between human health and environmental factors. Disaster risk management involves a process of anticipating, planning, and preparing for a response, with actions from different areas of the government, such as the health sector. This experience report had the objective of describing the actions developed by the environmental health surveillance of the Minas Gerais State Department of Health in response to the rupture of the B1 dam in Brumadinho. Among the actions developed, the monitoring of the quality of water for human consumption in the affected municipalities, the participation in meetings in spaces for decision and deliberation, and the production of technical documents are highlighted. The experiences lived by the team, during the disaster response actions, motivated reflections on the need to strengthen the development of the actions in an intra and intersectoral way, as well as the importance of qualifying the data recorded in the information systems, and knowledge of the local information of each territory, in order to subsidize the performance of the health sector in a timely manner in disaster situations.
\end{abstract}

KEYWORDS Public health surveillance. Man-made disasters. Environmental health. 


\section{Introdução}

A partir dos debates ocorridos na VIII Conferência Nacional de Saúde (1986), adota-se o conceito ampliado de saúde, que a define como resultante de um conjunto de condições, entre elas, o meio ambiente. A vigilância em saúde é considerada uma das funções essenciais da saúde pública, prevista como competência do Sistema Único de Saúde (SUS) na Constituição Federal de 1988 e na Lei no 8.080, de 1990, responsável pela informação para a ação e a intervenção que reduzam riscos e promovam a saúde nos territórios, considerando os complexos fenômenos econômicos, ambientais, sociais e biológicos que determinam o nível e a qualidade da saúde da população. A Lei no 8.080/1990 também reafirma a relação entre saúde e ambiente, uma vez que estabelece que o meio ambiente e o saneamento básico são fatores determinantes e condicionantes da saúde, incluindo como um dos campos de atuação do SUS, a colaboração na proteção do meio ambiente, bem como o controle da água para consumo humano e de substâncias tóxicas e radioativas'.

A vigilância em saúde ambiental é definida como um,

[...] conjunto de ações e serviços que proporcionam o conhecimento e a detecção de mudanças nos fatores determinantes e condicionantes do meio ambiente que interferem na saúde humana, com a finalidade de identificar, recomendar e adotar medidas de promoção da saúde ambiental, prevenção e controle dos fatores de risco relacionados às doenças ou a outros agravos à saúde2(45).

Pela Instrução Normativa 01/SVS, de 7 de março de 2005, dentre os fatores, destacam-se a água para consumo humano, os desastres naturais e os acidentes com produtos perigosos ${ }^{2}$. Dessa forma, a saúde ambiental se constitui o campo da saúde pública responsável pelas políticas públicas relacionadas com a interação entre a saúde humana e os fatores do meio ambiente, antrópicos e/ou naturais ${ }^{2}$.
A gestão de risco de desastres envolve um processo de antecipação, planejamento e preparação para resposta, com atuação de diferentes setores e esferas de governo nos âmbitos municipal, estadual e federal, assim como a sociedade organizada e as comunidades suscetíveis. O setor saúde tem grande responsabilidade nesse processo, uma vez que os impactos dos desastres resultam em efeitos diretos de curto, médio e longo prazos e indiretos sobre a saúde da população ${ }^{3}$.

A ocorrência de desastres tecnológicos, ocasionados pela intervenção do homem na natureza e pelo desenvolvimento tecnológico, ganhou destaque no Brasil, nos últimos anos, devido à sua magnitude. Como exemplos, destacam-se os rompimentos de barragens de mineração em Minas Gerais ocorridos em 2015 em Mariana e em 2019 em Brumadinho. Esses desastres caracterizam-se como situações de emergência em saúde pública e têm grandes impactos que extrapolam os municípios de ocorrência, incluindo impactos ambientais com destaque para contaminação de mananciais, degradação da qualidade da água bruta e, consequentemente, da qualidade da água para consumo humano que podem ocorrer em longo prazo, isto é, além do período imediato pós-desastre. Além disso, geram alteração abrupta da organização social e dos modos de viver e trabalhar historicamente constituídos nos territórios, com efeitos sobre a saúde da população ${ }^{4}$.

Observa-se, portanto grande interface entre as ações desenvolvidas pela Vigilância em Saúde Ambiental dos riscos associados aos desastres (Vigidesastres) e as ações do Programa Nacional de Vigilância da Qualidade da Água para Consumo Humano (Vigiagua).

Dessa forma, este relato de experiência tem o objetivo de descrever as ações desenvolvidas pela vigilância em saúde ambiental da Secretaria de Estado de Saúde de Minas Gerais (SES-MG) em resposta ao rompimento da barragem B1 em Brumadinho. 


\section{Material e métodos}

Este estudo consiste na descrição das principais ações desenvolvidas no âmbito da SES-MG pela equipe da Coordenação de Fatores de Risco Não Biológicos (CVFRNB), estruturadas em cinco grandes eixos.

O primeiro apresenta uma breve caracterização do desastre e do corpo hídrico atingido. $\mathrm{O}$ segundo se refere à caracterização da vigilância ambiental na SES-MG de acordo com a estrutura orgânica, composição da equipe e atividades desenvolvidas. $O$ terceiro descreve o monitoramento da qualidade da água para consumo humano. O quarto é composto por informações sobre reuniões em espaços de decisão e deliberação nas quais houve participação da equipe da CVFRNB. O quinto menciona os documentos técnicos produzidos para orientação das Unidades Regionais de Saúde e outros atores envolvidos nas atividades.

Espera-se que a experiência da vigilância em saúde ambiental do estado de Minas Gerais contribua para subsidiar a atuação de outros estados e municípios em situações de desastres ambientais.

\section{Caracterização do desastre e cenário atingido}

A barragem de rejeitos de mineração $\mathrm{B} 1$ do Complexo da Mina Córrego do Feijão localizava-se no município de Brumadinho, na Região Metropolitana de Belo Horizonte, entre as coordenadas $20^{\circ} 07^{\prime} 07^{\prime \prime}$ " $\mathrm{S}$ e $44^{\circ} 07^{\prime} 13^{\prime \prime} \mathrm{O}$, ocupando a área total de aproximadamente 27 hectares, com 87 metros de altura.

O município de Brumadinho está localizado a aproximadamente $19 \mathrm{~km}$ de Belo Horizonte e possui como municípios limítrofes: Igarapé, São Joaquim de Bicas, Mário Campos, Sarzedo, Ibirité, Belo Horizonte, Nova Lima, Itabirito, Moeda, Belo Vale, Bonfim, Rio Manso e Itatiaiuçu (figura 1).

Figura 1. Mapa de Brumadinho e municípios limítrofes, Minas Gerais
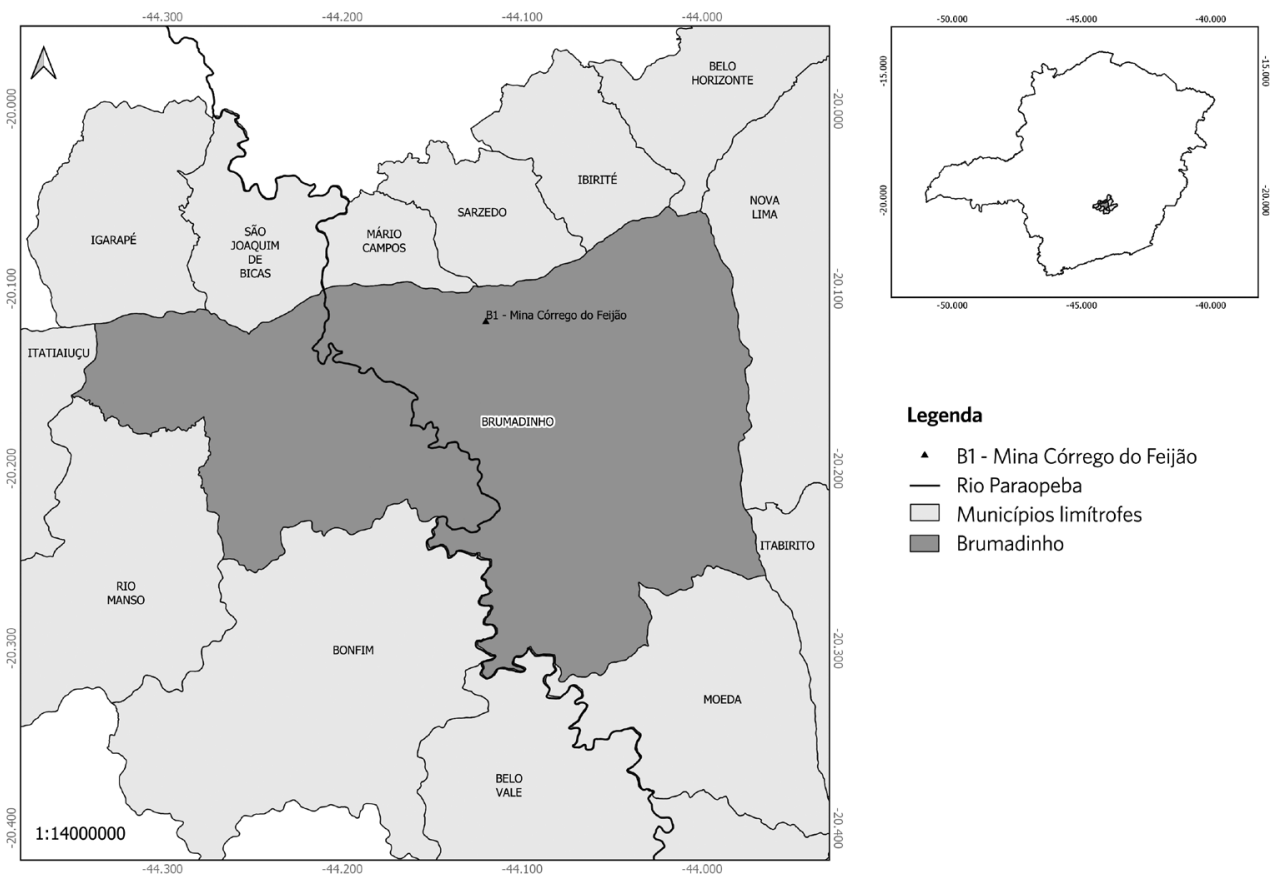

Legenda

- B1 - Mina Córrego do Feijão

- Rio Paraopeba

$\square$ Municípios limítrofes

$\square$ Brumadinho 
$\mathrm{O}$ acesso à barragem $\mathrm{B} 1$ pode ser realizado pelas vias BR-381, BR-262 e pela rodovia MG-040. Posteriormente a essas rodovias o acesso é realizado pelas estradas Alberto Flores e José Ribeiro Filho.

De acordo com o Instituto Mineiro de Gestão das Águas (Igam), a barragem B1 situa-se na Região Hidrográfica do Rio São Francisco, na bacia do ribeirão Ferro-Carvão, afluente do rio Paraopeba. A Bacia Hidrográfica do Rio Paraopeba possui como principal corpo d'agua o rio Paraopeba, que é um dos principais tributários do rio São Francisco, sendo sua nascente localizada no município de Cristiano Otoni, e sua foz, na represa de Três Marias. O território delimitado pela bacia é ocupado por 48 municípios, sendo que 35 possuem sedes urbanas no limite da bacia hidrográfica?.

Figura 2. Sub-bacia Hidrográfica do Rio Paraopeba, Minas Gerais, 2019

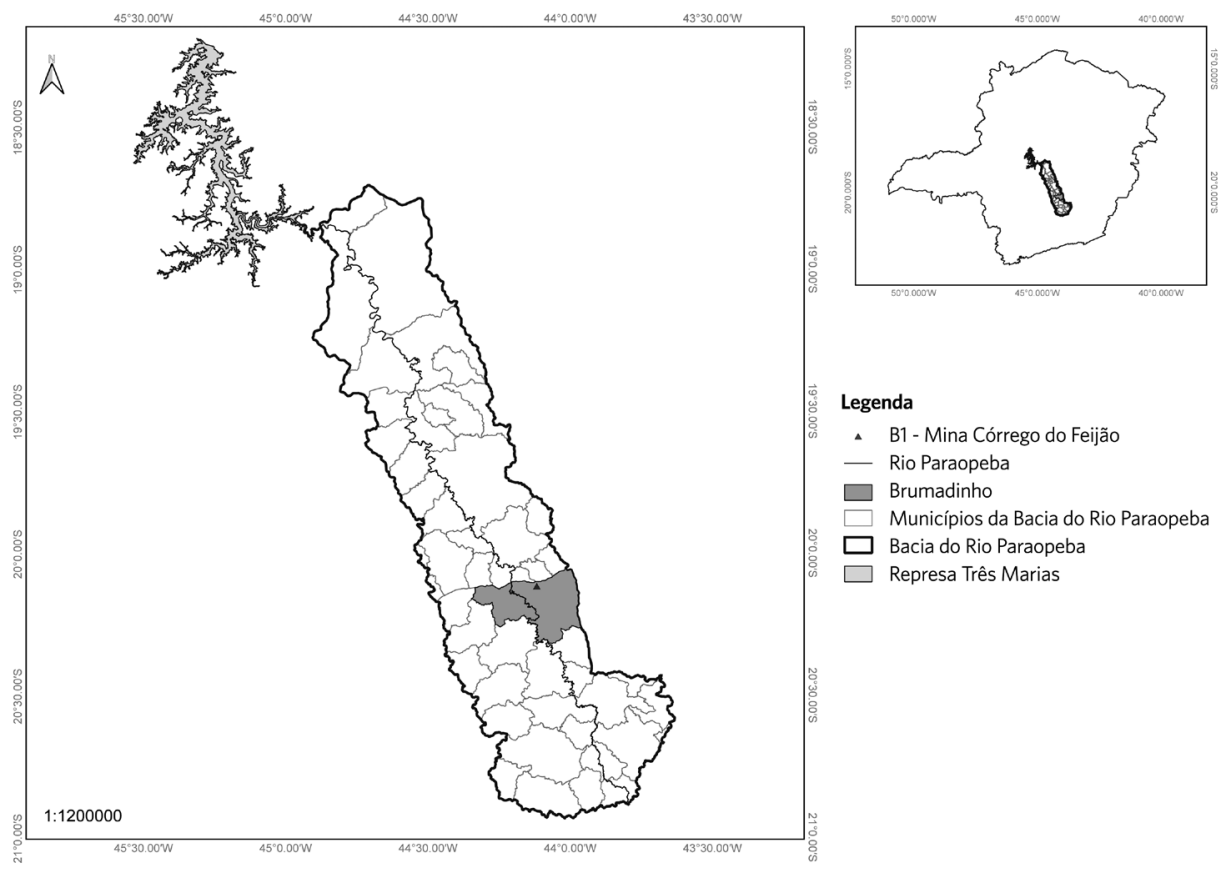

Fonte: Instituto Brasileiro de Geografia e Estatística. Sistema Estadual de Meio Ambiente e Recursos Hídricos 5,6.

Destaca-se que a Bacia Hidrográfica do Rio Paraopeba apresenta papel significativo no abastecimento de água para consumo humano da Região Metropolitana de Belo Horizonte, sendo que três de seus afluentes possuem em suas bacias reservatórios utilizados para esse abastecimento: rio Manso (Manso), ribeirão Juatuba (Serra Azul) e rio Betim (Várzea das Flores) ${ }^{\mathbf{8}}$.

Segundo informações da Companhia de Pesquisa de Recursos Minerais (CPMR Serviço Geológico do Brasil), os principais usos encontrados na bacia do rio Paraopeba estão relacionados com atividades minerárias, industrial, irrigação, abastecimento urbano e rural e geração de energia?.

O Complexo da Mina Córrego do Feijão está situado no Quadrilátero Ferrífero, região que representa uma área de cerca de 7 mil km² que possui grande importância econômica para o estado, devido a grandes reservas de Ferro e Ouro.

No dia 25 de janeiro de 2019, entre $12 \mathrm{~h} 25$ e 13 horas, ocorreu o rompimento da barragem B1 no Complexo da Mina Córrego Feijão, 
pertencente à Mineradora Vale/S.A. no município de Brumadinho. Foram liberados cerca de 12 milhões de metros cúbicos de rejeitos de produção mineral, espalhando uma espessa massa de rejeitos, que gerou perdas humanas e materiais ${ }^{10}$. De acordo com informações da Defesa Civil de Minas Gerais, em 4 de julho de 2019, entre as pessoas diretamente atingidas pelo desastre, houve 395 localizados, 23 desaparecidos e 247 óbitos ${ }^{11}$.

Foram atingidas as instalações de usina, o terminal de carregamento, as oficinas de manutenção e os prédios administrativos da mina do Córrego de Feijão, além de bloqueios no acesso rodoviário da mina até o vilarejo Córrego do Feijão e o acesso da portaria até o trevo de Alberto Flores. Pode-se considerar que todo o município foi afetado, uma vez que houve restrição de vias de acesso, sobrecarga no transporte e trânsito da cidade, nos atendimentos de saúde, alteração do calendário de algumas instituições de ensino, falta de água e energia elétrica por períodos curtos em alguns pontos, aumento do fluxo de pessoas ${ }^{10}$.

A pluma de rejeitos atingiu o ribeirão FerroCarvão, até desaguar no rio Paraopeba, seguiu até a Usina Hidrelétrica de Retiro Baixo, localizada no município de Pompéu, onde encontra-se retida até o momento, conforme monitoramento realizado pelo Igam ${ }^{12}$. Ao longo do trecho do rio Paraopeba atingido pelos rejeitos, estão inseridos 20 municípios, pertencentes às Unidades Regionais de Saúde Belo Horizonte, Divinópolis e Sete Lagoas. Ressalta-se que três desses municípios (Belo Horizonte, Pará de Minas e Paraopeba) eram abastecidos parcial ou totalmente pelas águas do rio Paraopeba, e interromperam as atividades de captação logo após a ocorrência do evento.

\section{Caracterização da vigilância ambiental na Secretaria de Estado de Saúde de Minas Gerais}

Na SES-MG, de acordo com o Decreto $\mathrm{n}^{\circ}$ 45.812, de 14 de dezembro de 2011, a CVFRNB está vinculada à Diretoria de Vigilância
Ambiental - Superintendência de Vigilância Epidemiológica, Ambiental e Saúde do Trabalhador - Subsecretaria de Vigilância e Proteção à Saúde. Em 2019, a equipe da coordenação apresenta composição multidisciplinar, formada por profissionais das áreas de geologia, geografia, gestão ambiental, química, enfermagem e fonoaudiologia ${ }^{13}$.

A CVFRNB tem por objetivo o conhecimento e a detecção ou prevenção de qualquer mudança nos fatores determinantes e condicionantes do meio ambiente que interferem na saúde humana por meio de atividades de vigilância em saúde ambiental relacionadas com água para consumo humano, ar, solo e contaminantes ambientais de importância e repercussão para saúde pública, bem como dos riscos decorrentes dos desastres naturais, acidentes com produtos perigosos e outros eventos capazes de causar doenças e agravos à saúde humana. As ações são desenvolvidas com a finalidade de recomendar e adotar medidas de promoção da saúde ambiental, prevenção e controle dos fatores de riscos relacionados com as doenças e outros agravos à saúde.

As atividades são realizadas com interlocução e apoio as 28 Unidades Regionais de Saúde definidas pela Resolução SES n ${ }^{\circ}$ 0811, de 30 de dezembro de 2005, e apresentam interface intra e intersetorial relacionada com os programas estaduais de vigilância em fatores de riscos não biológicos da vigilância em saúde ambiental ${ }^{14}$.

Dentre os principais órgãos em que há interface, destacam-se: a Secretaria de Estado de Meio Ambiente e Desenvolvimento Sustentável (Semad) e seus órgão vinculados (Instituto Mineiro de Gestão das Águas, Fundação Estadual do Meio Ambiente); a Coordenadoria Estadual de Defesa Civil; a Agência Reguladora de Serviços de Abastecimento de Água e de Esgotamento Sanitário do Estado de Minas Gerais; a Companhia de Saneamento de Minas Gerais (Copasa); os Serviços de Saneamento Integrado do Norte e Nordeste de Minas Gerais S/A (Copanor) e demais concessionárias autônomas de abastecimento de água e esgoto; além de órgão relacionados com a política 
de saneamento, como a Secretaria de Estado de Desenvolvimento e Integração do Norte e Nordeste de Minas Gerais; a Secretaria de Estado de Planejamento e Gestão e a Secretaria de Cidades e Integração Regional.

\section{MONITORAMENTO DA QUALIDADE DA ÁGUA PARA CONSUMO HUMANO}

Em resposta ao rompimento da barragem $\mathrm{B} 1$, no intuito de avaliar o impacto da pluma de rejeitos, considerando o risco associado à mobilização e ao transporte dos contaminantes provenientes do rejeito até o manancial subterrâneo, foi iniciado, pelo SUS, o monitoramento da água para consumo humano de soluções alternativas subterrâneas nos municípios que são banhados pelo rio Paraopeba após a confluência com o córrego Ferro-Carvão até o município de Três Marias.

De acordo com as 'Diretrizes para a priorização de áreas com populações sob risco de exposição a contaminantes químicos', a distância da população em relação à área contaminada em metros é um dos parâmetros utilizados para a identificação de áreas com população exposta ou potencialmente exposta, sendo atribuídos de 1 a 15 pontos, por categorias, para distâncias até mil metros. Para esse monitoramento, que tem o objetivo de verificar a qualidade da água utilizada para consumo humano, foram identificadas as soluções alternativas coletivas e individuais, que captam em fontes subterrâneas, localizadas até 100 metros das margens do rio Paraopeba, atingido pelo rejeito proveniente do rompimento. Essa distância foi estabelecida em discussão conjunta entre a equipe da SES-MG e a equipe do Ministério da Saúde considerando as características de baixa mobilidade dos possíveis contaminantes presentes no rejeito.

Os profissionais de saúde de Fortuna de Minas, Igarapé, Maravilhas e Pequi não identificaram formas de abastecimento que atendessem a esses critérios; portanto, esses municípios não foram incluídos no monitoramento.

O planejamento dos procedimentos de amostragem dos pontos para o monitoramento da qualidade da água para consumo humano foi realizado considerando os seguintes critérios: a representatividade do local amostrado na extensão do curso hídrico; a logística de acesso às localidades, visto que são na maioria distantes, com espaçamentos demográficos e malhas viárias raras e deficientes; os recursos disponíveis, como profissionais no âmbito do setor saúde, principalmente dos municípios, para identificação e seleção dos pontos amostrados, realização das coletas, manuseio, acondicionamento e transporte das amostras.

Inicialmente, o monitoramento foi realizado nas soluções alternativas coletivas subterrâneas cadastradas pela Secretaria Municipal de Saúde de Brumadinho. Concomitantemente, foram realizados, nos demais municípios atingidos, o levantamento e a caracterização das comunidades e das respectivas populações cujas soluções alternativas coletivas e individuais de abastecimento de água para consumo humano apresentam suas captações localizadas a uma distância de até 100 metros das margens do rio Paraopeba. Essas informações foram utilizadas para subsidiar a amostragem dos pontos e elaboração do plano de monitoramento (tabela 1). 
Tabela 1. Informações do plano de monitoramento da qualidade da água para consumo humano em resposta ao rompimento da barragem B1 em Brumadinho por município, Minas Gerais, 2019

\begin{tabular}{|c|c|c|c|c|c|}
\hline Município & $\begin{array}{l}\text { Data de início } \\
\text { das coletas }\end{array}$ & $\begin{array}{r}\text { Pontos } \\
\text { incluidos no } \\
\text { monitoramento } \\
\text { inicial }\end{array}$ & $\begin{array}{r}\text { Pontos do plano } \\
\text { de amostragem } \\
\text { vigente }\end{array}$ & $\begin{array}{l}\text { Quantidade de } \\
\text { campanhas }\end{array}$ & $\begin{array}{r}\text { Quantitativo } \\
\text { de amostras } \\
\text { coletadas }^{\star}\end{array}$ \\
\hline Betim & 28/03/2019 & 7 & 7 & 17 & 108 \\
\hline Brumadinho & 29/01/2019 & 13 & 4 & 40 & 180 \\
\hline Curvelo & $13 / 03 / 2019$ & 5 & 5 & 19 & 82 \\
\hline Esmeraldas & $21 / 02 / 2019$ & 13 & 9 & 22 & 180 \\
\hline Felixlândia & $14 / 03 / 2019$ & 6 & 5 & 19 & 68 \\
\hline Florestal & 28/03/2019 & 7 & 5 & 17 & 90 \\
\hline Juatuba & 22/02/2019 & 5 & 5 & 22 & 104 \\
\hline Mario Campos & 28/03/2019 & 11 & 5 & 17 & 59 \\
\hline Morada Nova de Minas & $14 / 03 / 2019$ & 5 & 5 & 19 & 80 \\
\hline Papagaios & $14 / 03 / 2019$ & 5 & 5 & 19 & 90 \\
\hline Pará de Minas & $14 / 03 / 2019$ & 18 & 6 & 18 & 89 \\
\hline Paraopeba & 28/02/2019 & 4 & 3 & 21 & 51 \\
\hline Pompéu & $14 / 03 / 2019$ & 4 & 3 & 19 & 40 \\
\hline São Joaquim de Bicas & 28/03/2019 & 11 & 8 & 17 & 100 \\
\hline São José da Varginha & $14 / 03 / 2019$ & 14 & 10 & 18 & 168 \\
\hline Três Marias & 14/03/2019 & 6 & 6 & 19 & 104 \\
\hline Total & - & 134 & 91 & 323 & 1593 \\
\hline
\end{tabular}

Fonte: Elaboração própria.

*Informações referentes a 30/10/2019.

A frequência adotada nesse monitoramento para avaliar os riscos decorrentes do rompimento da barragem B1 em Brumadinho é superior ao estabelecido para as ações de rotina, uma vez que, em situações de emergências em saúde pública, o monitoramento da qualidade da água pode ser ampliado de forma emergencial; e novos parâmetros ou agentes específicos, mesmo que não componham o padrão de potabilidade vigente, podem ser analisados com vistas a identificar sua presença na água ${ }^{15}$.

As coletas de amostras de água para esse monitoramento são realizadas por profissionais de saúde do SUS, referências técnicas das Unidades Regionais de Saúde (Belo Horizonte, Divinópolis e Sete Lagoas) e referencias técnicas de vigilância em saúde ambiental dos municípios. As coletas foram iniciadas em 29 de janeiro de 2019 em Brumadinho e encaminhadas para análise do Laboratório Central de Saúde Pública da Fundação Ezequiel Dias (Funed). Complementarmente, a partir de 21 de fevereiro de 2019, houve a contratação de laboratório realizada pela Vale S.A., em atendimento à decisão da Justiça Federal após audiência com representantes da Advocacia Geral da União e Ministério da Saúde, para realização de análises dos demais municípios atingidos, incluindo alguns pontos do plano de amostragem de Brumadinho.

No início do monitoramento, a periodicidade das coletas em cada forma de abastecimento era semanal; e, a partir do mês de abril, as coletas passaram a ser realizadas a cada 15 dias (tabela 1).

O plano de amostragem é constantemente reavaliado pelas equipes de vigilância em 
saúde com realização das adequações necessárias durante a execução das atividades. Houve a inclusão de pontos identificados após o início do monitoramento que também se enquadram nos critérios técnicos previamente definidos. Foram excluídos pontos que não cumpriam os critérios, como distância superior a 100 metros da margem do rio após conferência por geoprocessamento. Além disso, foram registradas intercorrências que motivaram a troca de pontos ou que inviabilizaram a coleta em datas pontuais, como recusas por parte de moradores para receber os técnicos da saúde no momento da coleta, poços que secaram devido ao período de estiagem, e falhas no funcionamento da bomba dos poços (tabela 1).

O quadro 1 apresenta os parâmetros analisados em cada amostra e os respectivos Valores Máximos Permitidos (VMP), estabelecidos no anexo XX da Portaria de Consolidação $\mathrm{n}^{\mathrm{o}}$ 5, de 28 de setembro de 2017 (quadro 1). Destaca-se que esses parâmetros e VMP integram o padrão de potabilidade e, por definição, devem ser observados na água para consumo humano ${ }^{16}$. Embora as coletas tenham sido realizadas em poços, reconhece-se que, na quase totalidade, eles são utilizados para consumo humano sem tratamento, portanto, procedeu-se à comparação dos resultados obtidos com o padrão de potabilidade.

Quadro 1. Parâmetros analisados no plano de monitoramento da qualidade da água para consumo humano em resposta ao rompimento da barragem B1 em Brumadinho e valores de referência

\begin{tabular}{|c|c|c|}
\hline Parâmetro & Unidade & Valor Máximo Permitido \\
\hline Alumínio Total & $\mathrm{mg} \mathrm{Al} / \mathrm{L}$ & 0,2 \\
\hline Antimônio Total & $\mathrm{mg} / \mathrm{L}$ & 0,005 \\
\hline Arsênio Total & $\mathrm{mg} / \mathrm{L}$ & 0,01 \\
\hline Bário Total & $\mathrm{mg} \mathrm{Ba} / \mathrm{L}$ & 0,7 \\
\hline Cádmio Total & $\mathrm{mg} \mathrm{Cd} / \mathrm{L}$ & 0,005 \\
\hline Chumbo Total & $\mathrm{mg} \mathrm{Pb} / \mathrm{L}$ & 0,01 \\
\hline Cobre Total & $\mathrm{mg} \mathrm{Cu} / \mathrm{L}$ & 2 \\
\hline Coliformes Totais (Qualitativo) & - & Ausente \\
\hline Cor Aparente & $\mathrm{mg} P t / L$ & 15 \\
\hline Cromo Total & $\mathrm{mg} \mathrm{Cr} / \mathrm{L}$ & 0,05 \\
\hline Escherichia coli (Qualitativo) & - & Ausente \\
\hline Ferro Total & $\mathrm{mg} \mathrm{Fe} / \mathrm{L}$ & 0,3 \\
\hline Manganês Total & $\mathrm{mg} \mathrm{Mn/L}$ & 0,1 \\
\hline Mercúrio Total & $\mathrm{mg} / \mathrm{L}$ & 0,001 \\
\hline Níquel Total & $\mathrm{mg} \mathrm{Ni} / \mathrm{L}$ & 0,07 \\
\hline $\mathrm{pH}$ & - & $6-9,5$ \\
\hline $\mathrm{pH}$ In Situ & - & $6-9,0$ \\
\hline Selênio Total & $\mathrm{mg} / \mathrm{L}$ & 0,01 \\
\hline Temperatura da Amostra & $\stackrel{\circ}{C}$ & - \\
\hline Temperatura do Ar & $\stackrel{\circ}{C}$ & - \\
\hline Turbidez & NTU & 5 \\
\hline Zinco Total & mg Zn/L & 5 \\
\hline
\end{tabular}

Fonte: Anexo XX da Portaria de Consolidação no 5, de 28 de setembro de 201716 
Os resultados são reportados, pelas Secretarias Municipais de Saúde, diretamente ao proprietário da localidade que teve sua forma de abastecimento de água para consumo humano monitorada. As famílias são orientadas pelas equipes de saúde, com apoio da equipe de Vigilância da Qualidade da Água para Consumo Humano, que, independentemente dos resultados das análises (satisfatórios ou insatisfatórios), fica mantida a medida cautelar inicial de suspensão da utilização da água das soluções alternativas individuais e coletivas (poços e cisternas) para consumo humano localizadas na área de risco definida (até 100 metros da margem do rio) conforme recomendação conjuntas da Secretaria de Estado de Saúde; da Semad; e da Secretaria de Estado de Agricultura, Pecuária e Abastecimento (Seapa) ${ }^{\mathbf{1 7}}$.

A partir dos resultados do monitoramento, pretende-se verificar a qualidade da água utilizada para consumo humano, tendo como objetivo caracterizar essas formas de abastecimento e avaliar os riscos à saúde, subsidiando adoção de medidas de prevenção e promoção da saúde. Foi realizada reunião técnica com as Unidades Regionais de Saúde de Belo Horizonte, Divinópolis e Sete Lagoas para caracterização dos pontos de monitoramento e alinhamento para discussão com equipe do Ministério da Saúde sobre as medidas que deverão ser adotadas de acordo com os cenários identificados, considerando os resultados do primeiro trimestre de monitoramento. Além disso, em janeiro de 2020, a SES-MG publicou o Boletim Informativo - Monitoramento da qualidade da água para consumo humano nos municípios atingidos pelo desastre da Vale/S.A., município de Brumadinho, Minas Gerais, com análise dos resultados para informar a população sobre a qualidade da água para consumo humano das formas de abastecimento monitoradas. Os resultados desse monitoramento da qualidade da água para consumo humano também foram apresentados pelo Ministério da Saúde, na edição especial de janeiro de 2020, no boletim epidemiológico
- Um ano de desastre da Vale - Organização e Resposta do Ministério da Saúde.

Este relato de experiência abrange da data da ocorrência do desastre até o dia 30 de outubro de 2019. Destaca-se que, em março de 2020, o monitoramento está em andamento com frequência quinzenal e será realizado até que o conjunto dos resultados possa subsidiar a decisão de sua interrupção.

Devido à possibilidade de carreamento de metais para as águas subterrâneas, principalmente durante o período chuvoso, o monitoramento das formas de abastecimento continua, bem como a avaliação periódica de seus resultados para auxiliar na tomada de decisão do setor saúde.

A partir da análise da série histórica e a depender dos resultados identificados nas amostras coletadas, algumas formas de abastecimento poderão voltar a ser utilizadas para consumo humano sem risco para a saúde, enquanto outras deverão passar por processo de tratamento adequado para ofertar uma água segura.

\section{Participação em espaços de deliberação e decisão}

Para subsidiar a tomada de decisões e deliberações visando à resposta imediata ao rompimento da barragem B1, da Mina Córrego do Feijão da Vale em Brumadinho, foi estabelecido, em 25 de janeiro de 2019, no município da ocorrência, o Posto de Comando, coordenado pela Coordenadoria Estadual de Defesa Civil de Minas Gerais (Cedec/MG), com a participação de órgãos e agências envolvidos nas ações de resposta ao desastre, entre eles, a SES-MG.

Concomitantemente, foi ativado o Comitê de Operações de Emergência na Saúde (Coes) da SES-MG, no dia 25 de janeiro de 2019, que integrou as áreas essenciais à resposta e emergência: Subsecretaria de Políticas e Ações de Saúde; Subsecretaria de Regulação do Acesso a Serviços e Insumos de Saúde; Subsecretaria de Vigilância e Proteção à Saúde; Subsecretaria de Inovação e Logística em Saúde e Subsecretaria de Gestão Regional. A 
participação ativa de representantes das áreas técnicas nesses espaços contribuiu para que a tomada de decisão ocorresse de forma mais ágil e eficiente.

Incialmente, as reuniões eram diárias; e, conforme a demanda, a frequência foi reduzida até sua desativação. No âmbito do Ministério da Saúde e da Secretaria Municipal de Saúde, também houve a ativação do Coes. Destacase que havia comunicação diária entres os comitês, com atualização da situação da emergência, identificação de demandas e andamento de ações identificadas como necessárias.

Paralelamente a esses espaços, foram realizadas reuniões técnicas entre a Secretarias de Estado de Saúde, a Semad e a Seapa, o Instituto Mineiro de Gestão das Águas e a Copasa, a fim de avaliar as ações que deveriam ser tomadas em relação a possível contaminação do rio Paraopeba, implicações em seus múltiplos usos, como consumo humano, irrigação e dessedentação animal, além de avaliar os dados que estavam sendo obtidos no monitoramento da qualidade da água.

No dia 26 de fevereiro de 2019, por meio do Decreto com numeração especial $n^{\circ} 176$, foi criado o Comitê Gestor Pró-Brumadinho, que reúne vários órgãos do governo do estado de Minas Gerais e que tem como objetivo coordenar as ações de recuperação, mitigação e compensação dos danos causados pelo rompimento da barragem B1 da Vale. Essa estrutura garante o efetivo compartilhamento de informações das ações em desenvolvimento e promove a sinergia entre os órgãos envolvidos ${ }^{18}$.

\section{Produção de documentos}

Como forma de subsidiar a atuação dos profissionais de saúde das Unidades Regionais de Saúde e das Secretarias Municipais de Saúde dos municípios atingidos pelo rompimento da barragem B1 em Brumadinho e de estabelecer diretrizes para ações de reparação desenvolvidas pela Vale S.A., foram elaborados formulários, documentos técnicos e notas informativas descritos a seguir:
1. Bases mínimas: orientações para o fornecimento de água para consumo humano aos grupos populacionais dos municípios atingidos pelo rompimento da barragem $\mathrm{B} 1$ da Mina Córrego do Feijão, da Vale S.A., em Brumadinho, em 25 de janeiro de 2019.

2. Nota Técnica ${ }^{\circ}$ 3/SES/SUBVPS-SVEASTDVA-CVFRNB/2019: plano emergencial de monitoramento da qualidade da água para consumo humano para os municípios atingidos pelo rompimento da barragem $\mathrm{B} 1 \mathrm{da}$ Mina Córrego do Feijão, da Vale S.A., em Brumadinho, em 25 de janeiro de 2019.

3. Nota Técnica ${ }^{\circ} 4 / \mathrm{SES} /$ SUBVPS-SVEASTDVA-CVFRNB/2019: abordagem com as famílias para avaliação de risco das formas de abastecimento de água para consumo humano próximas ao rio Paraopeba, área afetada pelo rompimento da barragem da Mina Córrego do Feijão da Vale S.A. em Brumadinho.

4. Formulário para identificação do consumo de água nos municípios atingidos pelo rompimento da barragem B1 da Mina Córrego do Feijão, da Vale S.A., em Brumadinho, em 25 de janeiro de 2019.

5. Planilha padronizada para consolidar o levantamento das formas de abastecimento próximas em até 100 metros do rio Paraopeba nos municípios a jusante do ponto de confluência entre o rejeito e o rio Paraopeba.

6. Ofício para as prestadoras de abastecimento de água para consumo humano (Copasa, Copanor), solicitando envio semanal dos laudos de análises do ponto de captação no rio Paraopeba a jusante do ponto de confluência com o córrego Ferro-Carvão.

7. Notas informativas para população e imprensa com orientações sobre cuidados necessários com a lama e rejeitos provenientes do rompimento da barragem de Brumadinho, 
*Orcid (Open Researcher and Contributor ID). divulgadas no site: http://www.saude.mg.gov. br/brumadinho.

8. Notas informativas conjuntas SES/Semad/ Seapa para população e impressa com orientações sobre cuidados necessários com a água do rio Paraopeba, divulgadas no site: http:// www.saude.mg.gov.br/brumadinho.

9. Nota técnica conjunta SES/Igam $n^{0} 1$ para a análise da qualidade das águas do rio Paraopeba a jusante da barragem B1 após o rompimento da barragem Bl operada pela mineradora Vale.

10. Nota técnica conjunta SES/Igam $\mathrm{n}^{\circ} 2$ para a análise da qualidade das águas do rio Paraopeba após desastre na barragem B1.

11. Nota técnica conjunta SES/Igam $n^{0} 3$ para a análise da qualidade das águas do Rio Paraopeba após desastre na barragem B1.

12. Boletim Informativo: Monitoramento da qualidade da água para consumo humano nos municípios atingidos pelo desastre da Vale/S.A., município de Brumadinho, Minas Gerais.

13. Brasil. Ministério da Saúde. Um ano do desastre da Vale: Organização e resposta do Ministério da Saúde. Bol Epidemiol. 2020; 51(n.esp.):1-35.

\section{Considerações finais}

A atuação da vigilância em saúde ambiental, em resposta ao rompimento da barragem $\mathrm{B} 1 \mathrm{em}$ Brumadinho, possibilitou o desenvolvimento de ações com importante articulação intrassetorial, com o envolvimento de profissionais de diferentes áreas do setor saúde dos municípios afetados, das unidades regionais de saúde e do nível central da SES-MG e do Ministério da Saúde. Além disso, destacam-se as parcerias intersetoriais realizadas com a Semad e seus órgãos vinculados (Instituto
Mineiro de Gestão das Águas, Fundação Estadual do Meio Ambiente); a Seapa; a Coordenadoria Estadual de Defesa Civil; o Corpo de Bombeiros Militar de Minas Gerais, essenciais para o planejamento e execução das ações que competem ao setor saúde.

A identificação e o mapeamento das soluções alternativas subterrâneas de água para consumo humano utilizadas pela população atingida pelo desastre foram desafios vivenciados durante esse processo, uma vez que a falta da atualização e a incompletude dessas informações no Sistema de Informação de Vigilância da Qualidade da Água para Consumo Humano (Sisagua) constitui uma fragilidade para a utilização dos dados. Dessa forma, foi necessária a realização de visitas in loco, o que aumenta a complexidade das ações realizadas e aumenta o tempo necessário para o desenvolvimento destas atividades.

As experiências vivenciadas pela equipe durante essa atuação motivaram reflexões sobre a necessidade de fortalecer o desenvolvimento das ações de forma intra e intersetorial, na rotina da vigilância em saúde ambiental e, principalmente, em situações de desastre uma vez que, em muitas atividades de trabalho desenvolvidas na rotina, observa-se a fragmentação ainda presente. Além disso, ressalta-se a importância de qualificação dos dados registrados no Sisagua e de conhecimento das informações locais de cada território na rotina de forma a subsidiar oportunamente, em situações de desastre, a identificação das formas de abastecimento de água para consumo humano e a elaboração de plano de amostragem emergencial para o monitoramento da qualidade da água.

\section{Colaboradoras}

Carvalho APM (0000-0002-9068-9686)*, Marques GL (0000-0002-9324-7648)*, Cunha JR (0000-0002-5784-0305)*, Pereira RA (0000-0002-9483-6604)* e Oliveira TS (00000003-1436-2777)* contribuíram igualmente para a elaboração do manuscrito. 


\section{Referências}

1. Brasil. Lei $\mathrm{n}^{\mathrm{o}}$ 8.080, de 19 de setembro de 1990. Dispõe sobre as condições para a promoção, proteção e recuperação da saúde, a organização e o funcionamento dos serviços correspondentes e dá outras providências. Diário Oficial da União. 20 Set 2020.

2. Brasil. Ministério da Saúde. Instrução Normativa $n^{\circ}$ 01, de 7 de março de 2005. Regulamenta a Portaria $\mathrm{n}^{\mathrm{o}} 1.172 / 2004 / \mathrm{GM}$, no que se refere às competências da União, estados, municípios e Distrito Federal na área de vigilância em saúde ambiental. Brasília, DF: Ministério da Saúde, 2005. Diário Oficial da União. 8 Mar 2005.

3. Brasil. Ministério da Saúde, Fundação Oswaldo Cruz. Guia de preparação e respostas do setor saúde aos desastres. [internet]. Rio de Janeiro: Fiocruz; MS; 2018. [acesso em 2019 set 20]. Disponível em: http:// www.ensp.fiocruz.br/portal-ensp/informe/site/arquivos/anexos/adbdf1fblbd20e237ab67233e3foa4cfe67a267c.PDF.

4. Freitas CM, Barcelos C, Asmus CIRF, et al. Da Samarco em Mariana à Vale em Brumadinho: desastres em barragens de mineração e Saúde Coletiva. Cad. Saúde Pública [internet]. 2019 [acesso em 2019 set 6]; 35(5):1-7. Disponível em: http://www.scielo.br/pdf/ csp/v35n5/1678-4464-csp-35-05-e00052519.pdf.

5. Instituto Brasileiro de Geografia e Estatística. [internet]. [Brasília, DF]: IBGE; [data desconhecida]. [acesso em 2019 set 27]. Disponível em: https://www.ibge. gov.br/geociencias/downloads-geociencias.html.

6. Minas Gerais. Secretaria de Estado de Meio Ambiente e Desenvolvimento Sustentável. Sistema Estadual de Meio Ambiente e Recursos Hídricos [internet]. [Belo Horizonte]: Sisema; [data desconhecida] [acesso em 2019 set 27]. Disponível em: http://idesisema. meioambiente.mg.gov.br/.

7. Instituto Mineiro de Gestão das Águas [internet]. [Belo Horizonte]: Igam; [data desconhecida]. [acesso em 2019 set 27]. Disponível em: http://www.igam. mg.gov.br/index.php?option=com_content $\&$ task=vi ew\&id=154\&Itemid=140.

8. Serviço Geológico do Brasil. Monitoramento Especial da Bacia do Rio Paraopeba. Relatório I: monitoramento hidrológico e sedimentométrico. [internet]. 2019. [acesso em 2019 set 6]. Disponível em: http://www. cprm.gov.br/sace/conteudo/paraopeba/RT_01_2019_ PARAOPEBA.pdf.

9. Serviço Geológico do Brasil. Monitoramento Especial da Bacia do Rio Paraopeba. Relatório II: monitoramento geoquímico. [internet]. 2019 mar. [acesso em 2019 set 6]. Disponível em: http://www.cprm.gov. br/sace/conteudo/paraopeba/RT_02_2019_PARAOPEBA.pdf.

10. Brasil. Ministério da Integração Nacional [internet]. [Brasília, DF]: MI; [data desconhecida] [acesso em 2019 set 27]. Disponível em: https://s2id-search.labtrans.ufsc.br/.

11. Minas Gerais. Gabinete Militar do Governador. Coordenadoria Estadual de Defesa Civil. [internet]. [Belo Horizonte]: CEDEC; [27 maio 2019]. [acesso em 2019 set 27]. Disponível em: http://www.defesacivil.mg.gov.br/index.php/component/gmg/page/669-Brumad_04_07_19.

12. Instituto Mineiro de Gestão das Águas. Informativo $\mathrm{n}^{0}$ 52: Informativo Mensal da qualidade das águas do Rio Paraopeba, após o desastre na barragem B1 no complexo da Mina Córrego Feijão da Mineradora Vale/SA no município de Brumadinho - Minas Gerais. [internet]. 2019. [acesso em 2019 set 6]. Disponível em: http://www.meioambiente.mg.gov.br/images/ stories/2019/DESASTRE_BARRAGEM_B1/informativos_qualidade_agua/Informativo_52_IGAM_COPASA_CPRM_final.pdf.

13. Minas Gerais. Secretaria de Estado de Saúde. Decreto $\mathrm{n}^{\circ} 45.812$, de 14 de dezembro de 2011. Dispõe sobre a organização da Secretaria de Estado de Saúde de Minas Gerais. [internet]. Diário Oficial de Minas 
Gerais. [acesso em 2019 set 27]. Disponível em: https://www.saude.mg.gov.br/images/documentos/Decreto\%2045.812_2011\%20-\%20dispoe\%20sobre\%20 a\%20organizacao\%20da\%20SES.pdf.

14. Minas Gerais. Secretaria de Estado de Saúde. Resolução SES no 0811, de 30 de dezembro de 2005. Identifica as Gerências Regionais de Saúde - GRS do Estado de Minas Gerais e estabelece as suas respectivas áreas de abrangência. [internet]. Diário Oficial de Minas Gerais. 31 Dez 2005. [acesso em 2019 set 27]. Disponível em: http://www.saude.mg.gov.br/images/ documentos/res_0811.pdf.

15. Brasil. Ministério da Saúde. Diretriz Nacional do Plano de Amostragem da Vigilância da Qualidade da Água para Consumo Humano [recurso eletrônico]. Brasília, DF: Ministério da Saúde; 2016.

16. Brasil. Ministério da Saúde. Portaria de Consolidação $n^{\circ} 4$, de 28 de setembro de 2017. Consolidação das normas sobre os sistemas e os subsistemas do Sistema Único de Saúde. Anexo XX - do controle e da vigilância da qualidade da água para consumo humano e seu padrão de (Origem: PRT MS/GM 2914/2011). Diário Oficial da União. 28 Set 2017.
17. Minas Gerais. Secretaria de Estado de Saúde de Minas Gerais. Suspensão do uso de água bruta é ampliada no Rio Paraopeba [internet]. [Belo Horizonte]: SES-MG; [22 fev 2019]. [acesso em 2019 set 27]. Disponível em: https://www.saude.mg.gov.br/component/ gmg/story/10921-suspensao-do-uso-de-agua-bruta-e-ampliada-no-rio-paraopeba.

18. Minas Gerais. Governo do Estado de Minas Gerais. Decreto NE no 176, de 26 de fevereiro de 2019. Institui o Comitê Gestor Pró-Brumadinho em decorrência da ruptura da Barragem I da Mina do Córrego do Feijão, no Município de Brumadinho. [internet]. Diário Oficial de Minas Gerais. 27 Fev 2019. [acesso em 2019 set 27]. Disponível em: http://jornal.iof.mg.gov.br/xmlui/bitstream/handle/123456789/214887/caderno1_2019-02-27\%201. pdf? sequence=1\#: :text=DECRETO $\% 20 \mathrm{NE} \% 20$ N\%C2\%BA\%20176\%2C\%20DE,o\%20inciso\%20 vII\%20do\%20art\%20.

Recebido em 27/09/2019

Aprovado em 22/04/2020

Conflito de interesses: inexistente

Suporte financeiro: não houve 